\title{
Adhesion and friction control localized folding in supported graphene
}

K. Zhang ${ }^{1}$ and M. Arroyo ${ }^{1, a)}$

LaCàN, Universitat Politècnica de Catalunya-BarcelonaTech (UPC), Barcelona 08034, Spain

(Dated: 23 April 2013)

Graphene deposited on planar surfaces often exhibits sharp and localized folds delimiting seemingly planar regions, as a result of compressive stresses transmitted by the substrate. Such folds alter the electronic and chemical properties of graphene, and therefore it is important to understand their emergence, to either suppress them or control their morphology. Here, we study the emergence of out-of-plane deformations in supported and laterally strained graphene with high-fidelity simulations and a simpler theoretical model. We characterize the onset of buckling and the nonlinear behavior after the instability in terms of the adhesion and frictional material parameters of the graphene-substrate interface. We find that localized folds evolve from a distributed wrinkling linear instability due to the nonlinearity in the van der Waals graphene-substrate interactions. We identify friction as a selection mechanism for the separation between folds, as the formation of far apart folds is penalized by the work of friction. Our systematic analysis is a first step towards strain engineering of supported graphene, and is applicable to other compressed thin elastic films weakly coupled to a substrate.

a)Electronic mail: marino.arroyo@upc.edu 


\section{INTRODUCTION}

Graphene is a single layer of carbon atoms densely packed in a two-dimensional hexagonal lattice. It has attracted considerable attention due to its exceptional structure, mechanical, chemical and electronic properties, which offer unique possibilities in nanostructured materials and devices. ${ }^{1-3}$ Deviations from a perfectly planar state are pervasive in supported and free-standing graphene. Indeed, at length-scales $\ell>100 h \sim 10 \mathrm{~nm}$, significantly larger than the nominal mechanical thickness, ${ }^{4}$ graphene is very easily bendable, conforms to a wavy substrate, ${ }^{5,6}$ and is prone to relaxing in-plane deformations by buckling outof-plane. Such deformations in turn strongly influence the electronic properties ${ }^{7-10}$ and

\section{the chemical reactivity, ${ }^{11}$ opening new possibilities for engineering such properties through} strain. ${ }^{12,13}$ Massive crumpling of few-layer graphene can also be controlled to tune the coating hydrophobicity. ${ }^{14}$ The emergence of ripples in free-standing graphene has been modeled

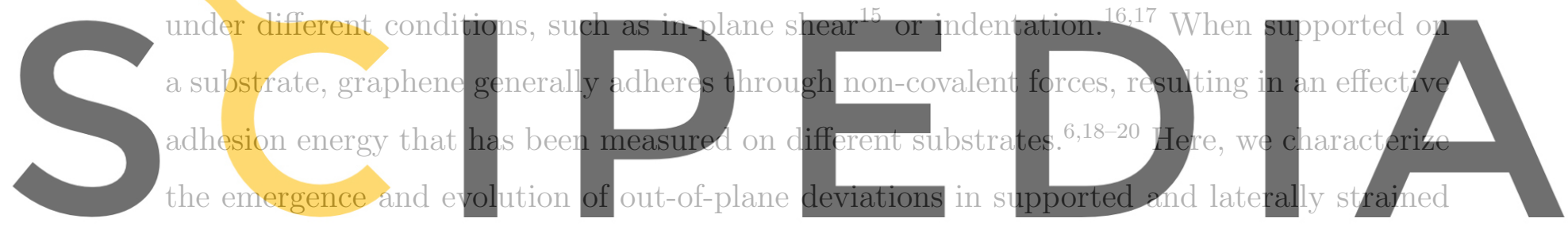

single layer graphene.

Register for free at https//www.scipedia.com to download the version without the watermark

Folds are commonly observed in single or few-layer graphene grown by chemical vapor

deposition $(\mathrm{CVD}),{ }^{21-23}$ although they can also be present in exfoliated graphene. ${ }^{10}$ in $\mathrm{CVD}$ grown graphene, the folds often form networks with a typical spacing between hundreds of nanometers and microns, and are attributed to the compressive stress arising upon cooling due to the differential thermal expansion between graphene and the metal substrate. ${ }^{24}$ There is evidence that new folds arise during transfer of CVD-grown graphene to a different substrate, that these folds strongly depend on the topography and polycrystalline structure of the growth substrate, and that depending on the transfer procedure folds can be either preserved or released. ${ }^{25}$ The network of folds has been controlled by patterning the topography of the growth substrate, ${ }^{22,26}$ by tuning the transfer method, ${ }^{27}$ or by straining the substrate. ${ }^{14}$

A number of laterally compressed systems consisting of an elastic film coupled to a substrate develop distributed sinusoidal wrinkles, which evolve upon further compression either by coarsening ${ }^{28,29}$ or by forming localized folds. ${ }^{30,31}$ Interestingly, creasing is a distinct localized surface instability in soft elastic solids that nucleates from a uniform state and precedes 
distributed wrinkling, ${ }^{32,33}$ although in the presence of a thin stiffer crust the wrinkle-to-fold sequence of events is recovered. ${ }^{34}$ Uniaxially compressed elastic films floating on a fluid have also been examined, ${ }^{35}$ reporting a curvature localization after a delocalized periodic wrinkling with a characteristic wavelength. In this system, the wrinkle-to-fold transition occurs when the film is compressed beyond a third of its initial wrinkle wavelength, and has been attributed to geometric nonlinearity. In a different context, a wrinkle-to-fold transition has been explained by the nonlinearity of a deformable substrate. ${ }^{36}$ Under biaxial compression, it has been demonstrated that the geometry and topology of the fold network can be controlled by the strain anisotropy and magnitude. ${ }^{34}$

When deposited on a soft substrate and compressed, graphene has been shown to deform together with the substrate surface to develop distributed wrinkling. ${ }^{37}$ Here we focus on stiffer substrates, whose surface is assumed to remain planar. Consequently, any out-ofplane deformation of graphene such as wrinkling involves delamination, mobilizing different

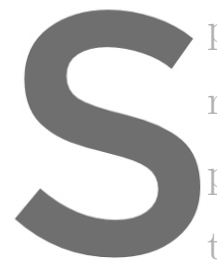
physics. In this situation, distributed wrinkles associated to compression have not been
reported. As discussed before by analogy to similar systems, localized folds are presumably
preceded by such wrinkles. Our goal is to understand the emergence of folds, to characterize
their spacing, and to examind auantitatively the effect of the mechanical counling betureen Register for free at https//WwWw.scipedia.com to download the version without the watermark substrate through non-bonded interactions, and therefore the spacing between folds cannot

be explained by nonlocal elastic fields in the substrate. ${ }^{36}$ instead, we hypothesize that friction is responsible for the relatively uniform fold separation observed in experiments, and include this effect in our model.

The rest of the paper is organized as follows. Section II describes the simulation methodology and provides a simple example of wrinkling and folding in supported graphene. Section III outlines the theoretical model for the emergence of wrinkling, the wrinkle-to-fold transition, and the selection principle for the separation between folds. The results and the summary and conclusions are given in Sections IV and V respectively.

\section{SIMULATION METHODOLOGY AND TYPICAL RESULT}

Over the last decade, it has been demonstrated that the mechanics of graphene and carbon nanotubes can be accurately described by continuum models in the full nonlinear 
regime in the absence of defects. ${ }^{38-42} \mathrm{~A}$ number of atomistic-based continuum approaches have been proposed. Here, we model graphene as a continuous surface without thickness, whose internal energy per unit underformed surface is given by a hyper-elastic potential $W(\boldsymbol{C}, \mathcal{K})$ that depends on the in-plane stretch (the metric tensor) of the surface $\boldsymbol{C}$ and its curvature (the second fundamental form) $\mathcal{K} .{ }^{43}$ The potential $W$ is systematically derived from the atomistic interactions describing the bonding energy and forces using a kinematic rule linking continuum and lattice deformations, the exponential Cauchy-Born rule, and averaging the atomistic energy in one unit cell of the lattice. This continuum model retains the crystal symmetry of the underlying graphene sheet. This model has been shown to very accurately mimic all-atom simulations exhibiting very large deformations and buckling instabilities. ${ }^{44}$ When this model is linearized around the planar ground state of graphene, the in-plane and bending elasticities are isotropic, and can be characterized by a surface Young's modulus $Y_{s}$ (with units of line tension), a Poisson ratio $\nu$, and a bending modulus
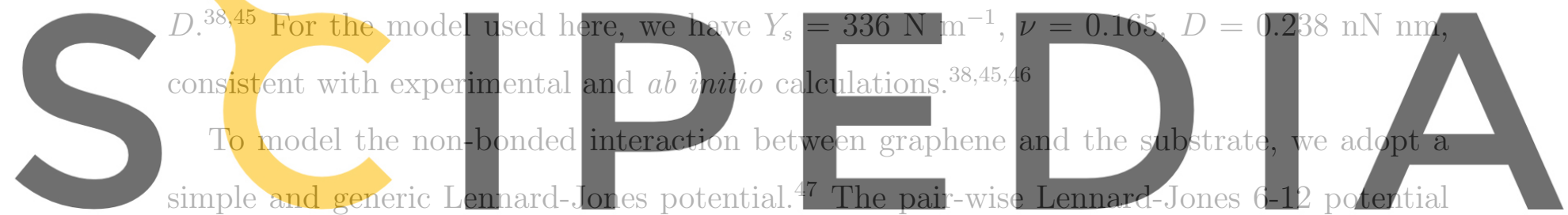

between a graphene and a substrate atom a distance $r$ apart is

Register for free at https//www.scipedia.com to download the version without the watermark

$$
V(r)=4 \epsilon\left[-\left(\frac{\sigma}{r}\right)^{6}+\left(\frac{\sigma}{r}\right)^{12}\right]
$$

where $x=\sqrt[6]{2} \sigma$ is the equilibrium distance at which the potential attains its minimum, and $\epsilon$ is the energy at the equilibrium distance. These atom-atom interactions result in an effective point-half space interaction energy (per unit surface area) of the form ${ }^{48}$

$$
\mathcal{V}(h)=-\gamma\left[\frac{3}{2}\left(\frac{h_{0}}{h}\right)^{3}-\frac{1}{2}\left(\frac{h_{0}}{h}\right)^{9}\right],
$$

where $h$ is the separation between a given point and the planar substrate, $h_{0}$ is the equilibrium separation between the elastic sheet and a half-space, and $\gamma$ denotes the well depth or adhesion energy.

Denoting by $\boldsymbol{x}$ a parametrization of the graphene sheet and orienting $x_{3}$ perpendicular to the substrate, the bonded and non-bonded energy of the graphene sheet adhered to the substrate can be written as

$$
U[\boldsymbol{x}]=\int_{\Omega_{0}} W(\boldsymbol{C}, \mathcal{K}) d S_{0}+\int_{\Omega_{0}} \mathcal{V}\left(x_{3}\right) d S_{0},
$$


where $\Omega_{0}$ is the reference domain of the graphene film, containing nominally a fixed number of atoms. Hence, we adopt a total Lagrangian formulation. ${ }^{44}$ Numerically, $\boldsymbol{x}$ is discretized with subdivision finite elements, ${ }^{49}$ which provide a smooth parametrization with square integrable curvature. The resulting energy function, depending on the coordinates of the finite element nodes, is minimized numerically with a quasi-Newton BFGS algorithm. ${ }^{44} \mathrm{We}$ implement periodic boundary conditions, and strain the sample by modifying the actual size of the periodic box, while the mass of material (the area of the reference domain $\Omega_{0}$ ) remains fixed.

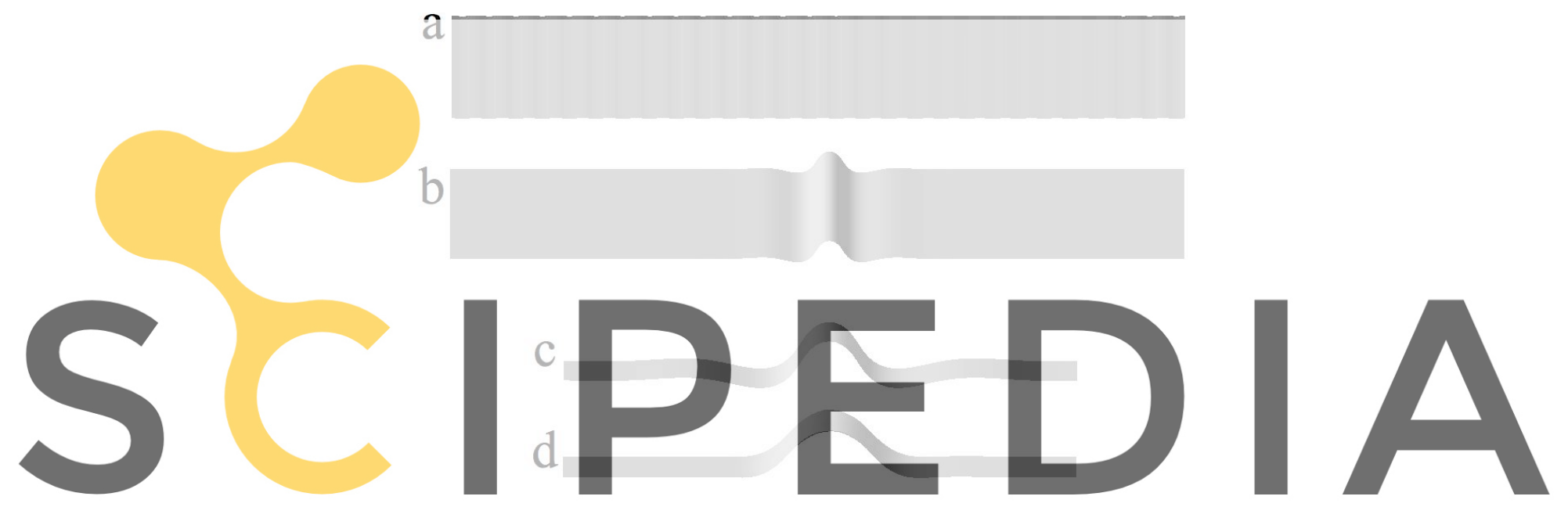

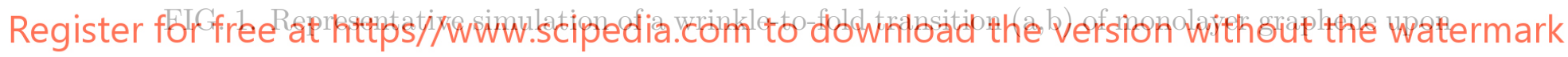

uniaxial compression. The length of the domain along the uniaxial compression is $200 \mathrm{~nm}$, the

adhesion energy is $0.45 \mathrm{~J} / \mathrm{m}^{2}$, and equilibrium separation of the potential is $6 \mathrm{~nm}$. A lateral view of the fold is given in (c), to be compared with the fold morphology obtained with a smaller equilibrium separation $\left(h_{0}=0.6 \mathrm{~nm}\right)$ shown in $(\mathrm{d})$.

Figure 1 shows a typical numerical simulation exhibiting wrinkling and folding. A periodic graphene slab with length $L_{0}=200 \mathrm{~nm}$ is uniaxially compressed by incrementally decreasing the periodic length along one coordinate by a factor, $L_{n}=f^{n} L_{0}$ with $0<f<1$, where in practice $f$ is close to one. Initially, the graphene slab stays planar until, beyond a threshold, it develops periodic, small amplitude wrinkles, Fig. 1(a). As in similar systems, ${ }^{35,50}$ the finite wavelength is set by a competition between bending energy, which penalizes high frequency undulations, and the interaction with the substrate, which penalizes large amplitude and low frequency out-of-plane deviations. By further compressing the sample, the out-of-plane deformation localizes into a single, sharp fold, Fig. 1(b). The details of this generic process, 
e.g. the critical strain or the wrinkling and folding morphology, strongly depend on the parameters of the adhesion potential, $h_{0}$ and $\gamma$. For instance, for large values of $h_{0}$, the folds are surrounded by regions of negative out-of-plane displacement, Fig. 1(c), absent for small $h_{0}$, Fig. 1(d).

\section{THEORETICAL MODEL}

\section{A. Emergence of wrinkling}

To understand the emergence of wrinkling and folding in supported graphene, we develop next a simple analytical model, largely following previous works on compressed thin films. ${ }^{51,52}$

We consider a rectangular graphene film under uniaxial compression. We denote by $u(x)$ and $w(x)$ the in-plane and the out-of-plane displacements of the film, and by $\varepsilon$ the uniaxial

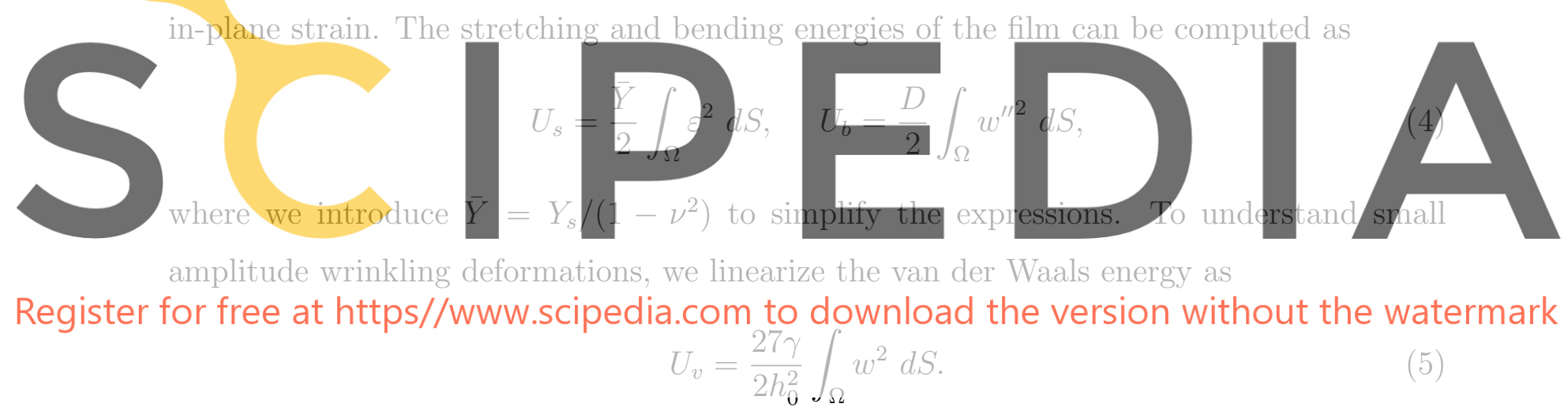

Adopting a von Karman nonlinear plate theory, ${ }^{53}$ the membrane strain of the film can be approximated as

$$
\varepsilon=\varepsilon_{0}+u^{\prime}+\frac{1}{2} w^{\prime 2}
$$

where $\varepsilon_{0}$ represents the globally applied lateral strain on the film. We consider the ansatz $w(x)=A \cos (k x)$ for the out-of-plane displacement. By requiring the uniformity of the in-plane tension or strain, see Eq. (6), the in-plane displacements should obey $u(x)=$ $(1 / 8) k^{2} A^{2} \sin (2 k x)$, and the constant strain becomes $\varepsilon=\varepsilon_{0}+(1 / 4) k^{2} A^{2} .{ }^{52}$ We note that the applied strain on the system, here $\varepsilon_{0}<0$, differs from the film membrane strain $\varepsilon<0$, which is partially relaxed by the positive term $(1 / 4) k^{2} A^{2}$. The stretching energy difference per unit area takes the form

$$
\Delta \bar{U}_{s}(A, k)=\frac{\bar{Y}}{2}\left(\varepsilon^{2}-\varepsilon_{0}^{2}\right)=\frac{T_{0}}{4} k^{2} A^{2}+\frac{\bar{Y}}{32} k^{4} A^{4},
$$


where $T_{0}=\bar{Y} \varepsilon_{0}$ is a reference surface tension (it is only the actual surface tension for a uniform planar state). The total energy difference per unit area then becomes

$$
\Delta \bar{U}(A, k)=\left(\frac{T_{0}}{4} k^{2}+\frac{D}{4} k^{4}+\frac{27 \gamma}{4 h_{0}^{2}}\right) A^{2}+\frac{\bar{Y}}{32} k^{4} A^{4},
$$

where the last term accounts for the stretching induced by wrinkling, and has a stabilizing effect.

The onset of wrinkling from the planar state $(A=0)$ can be established by the loss of stability condition $\partial^{2} \Delta \bar{U} / \partial A^{2}(0, k) \leq 0$, which leads to

$$
T_{0} \leq-D k^{2}-\frac{27 \gamma}{h_{0}^{2} k^{2}}
$$

The wavenumber of the most unstable mode can be found by maximizing the expression above (recall $T_{0}$ is negative) with respect to $k^{2}$, yielding

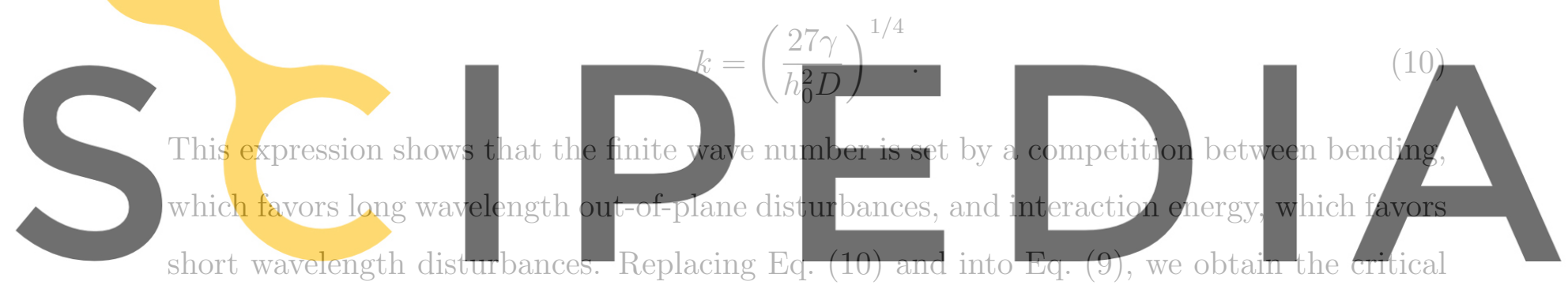

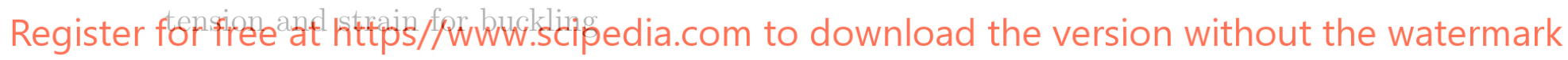

$$
T_{c r}=-\frac{2}{h_{0}} \sqrt{27 \gamma D}, \quad \varepsilon_{c r}=-\frac{2}{h_{0} \bar{Y}} \sqrt{27 \gamma D} \text {. }
$$

Minimizing the total energy density in Eq. (8) with respect to $A$, we find

$$
A=2 \sqrt{-\frac{1}{\bar{Y}}\left(T_{0} k^{-2}+D+\frac{27 \gamma}{h_{0}^{2}} k^{-4}\right)} .
$$

The expression under the square root is positive beyond the stability point, c.f. Eq. (9). Replacing the expression above in $\Delta \bar{U}(A, k)$ results in

$$
\Delta \bar{U}(k)=-\frac{1}{2 \bar{Y}}\left(T_{0}+D k^{2}+\frac{27 \gamma}{h_{0}^{2}} k^{-2}\right)^{2}
$$

Minimizing the energy with respect to the wave number, we recover Eq. (10) even beyond the critical strain, and obtain

$$
\Delta \bar{U}_{\min }=-\frac{1}{2 \bar{Y}}\left(T_{0}+2 \sqrt{\frac{27 \gamma D}{h_{0}^{2}}}\right)^{2}
$$


Replacing Eq. (10) into Eq. (12), we find

$$
A=\frac{\lambda}{\pi} \sqrt{\left(T_{c r}-T_{0}\right) / \bar{Y}}=\frac{\lambda}{\pi} \sqrt{\varepsilon_{c r}-\varepsilon_{0}},
$$

where $\lambda=2 \pi / k$ is the wavelength. We shall compare later these estimates with our fully nonlinear simulations.

\section{B. Wrinkle to fold transition}

Similarly to the previous treatment of wrinkling, we adopt the von Karman theory with an ansatz for the folding geometry in agreement with our simulations. We consider

$$
w(x)=A \cos \frac{\pi x}{l}+B \cos \frac{2 \pi x}{l}+A-B
$$

for $x \in[-l, l]$ and $w(x)=0$ otherwise, for $x \in(-L / 2,-l) \cup(l, L / 2)$. Here, $A$ and $B$ are amplitudes, $l$ is half of the fold length, and $I$ is the length of the graphene film The term

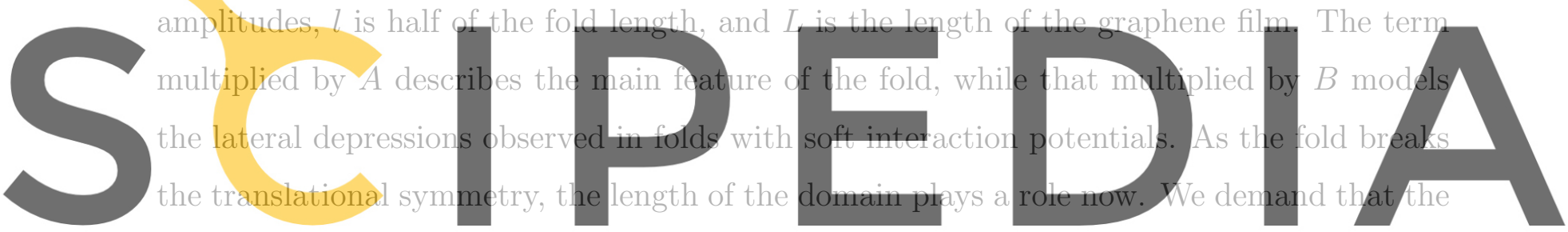

membrane tension (strain) be uniform, see Eq. (6), and solve for the in-plane displacement

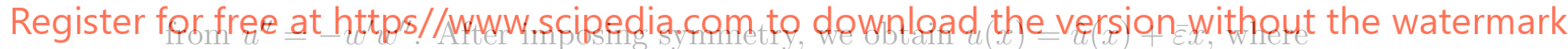

$$
\begin{aligned}
\hat{u}(x)= & \frac{\pi}{l}\left(\frac{A^{2}}{8} \sin \frac{2 \pi x}{l}+\frac{A B}{3} \sin \frac{3 \pi x}{l}-A B \sin \frac{\pi x}{l}\right. \\
& \left.+\frac{B^{2}}{4} \sin \frac{4 \pi x}{l}-\pi \frac{A^{2}+4 B^{2}}{4} \frac{x}{l}\right)
\end{aligned}
$$

in the interval $[-l, l]$. It is easy to see from Eq. (6) that in this interval $\varepsilon=\varepsilon_{0}+\bar{\varepsilon}$. Outside this interval, graphene remains planar with the same constant strain $\varepsilon$. To find $\bar{\varepsilon}$, we impose continuity of the in-plane displacement at $x= \pm l$, i.e.

$$
u( \pm l)=\mp(L / 2-l) \bar{\varepsilon},
$$

from which we obtain

$$
\bar{\varepsilon}=\frac{\pi^{2}}{2 L l}\left(A^{2}+4 B^{2}\right) .
$$

Thus, the stretching energy difference per unit area becomes

$$
\Delta \bar{U}_{s}(A, B, l)=T_{0} \bar{\varepsilon}+\frac{\bar{Y}}{2} \bar{\varepsilon}^{2} .
$$


A simple calculation shows that the bending energy density can be computed as

$$
\bar{U}_{b}(A, B, l)=\frac{D \pi^{4}}{2 L l^{3}}\left(A^{2}+16 B^{2}\right)
$$

We turn now to the graphene-substrate interaction. A harmonic approximation disregards the physics of folding, which is promoted by decohesion for large amplitude out-of-plane displacements of the film, beyond the inflection point of the potential. Also, the folding geometry is strongly affected by the stiffening of the interaction as the film is brought closer to the substrate. With a harmonic approximation, folding is never energetically preferable to wrinkling, from which we conclude that the nonlinearity of the graphenesubstrate interaction is crucial to explain the wrinkle-to-fold transition in this system. We find that the qualitative features of the wrinkle-to-fold transition can be captured with a third order expansion of the interaction potential. However, for accurate predictions of the model, we approximate the interaction potential with a five term expansion
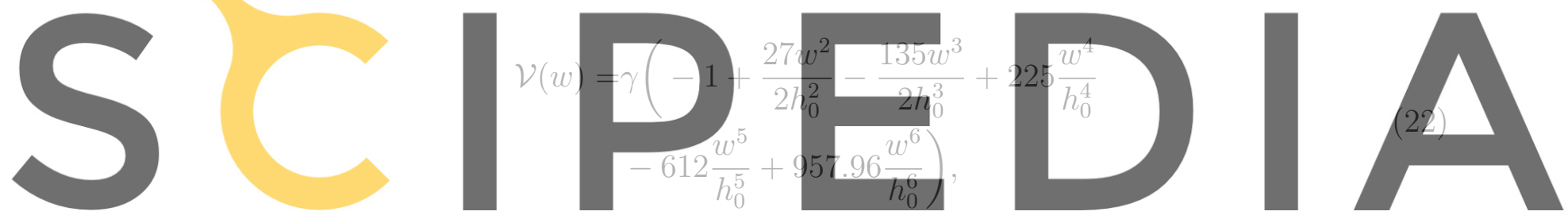

Register where the last term is modified to match the inflectipn point of the original potential. The for free at https//www.scipedia.com to download the version without the watermark corresponding energy density after integration is

$$
\begin{aligned}
\bar{U}_{v}(A, B, l)= & \frac{27 \gamma l}{2 h_{0}^{2} L}\left(3 A^{2}-4 A B+3 B^{2}\right)-\frac{135 \gamma l}{4 h_{0}^{3} L}\left(10 A^{3}-\right. \\
& \left.15 A^{2} B+18 A B^{2}-10 B^{3}\right)+\frac{225 \gamma l}{4 h_{0}^{4} L}\left(35 A^{4}-\right. \\
& \left.56 A^{3} B+84 A^{2} B^{2}-80 A B^{3}+35 B^{4}\right)- \\
& \frac{153 \gamma l}{h_{0}^{5} L}\left(63 A^{5}-105 A^{4} B+180 A^{3} B^{2}-\right. \\
& \left.225 A^{2} B^{3}+175 A B^{4}-63 B^{5}\right)+\frac{59.87 \gamma l}{h_{0}^{6} L} \\
& \left(462 A^{6}-792 A^{5} B+1485 A^{4} B^{2}-\right. \\
& \left.2200 A^{3} B^{3}+2310 A^{2} B^{4}-1512 A B^{5}+462 B^{6}\right) .
\end{aligned}
$$

The total energy density relative to a planar state under a strain $\varepsilon_{0}$ is

$$
\Delta \bar{U}(A, B, l)=\Delta \bar{U}_{s}+\bar{U}_{b}+\bar{U}_{v}
$$


We find candidate equilibrium states by minimizing the total energy with respect to $A, B$, and $l$ numerically. Depending on the applied strain, $\varepsilon_{0}$, we estimate the wrinkle-to-fold transition by comparing the energies of the optimal wrinkling pattern and of the optimal fold, as reported in the results section.

\section{Separation between folds: the role of friction}

In the previous model for folding, the length of the graphene film $L$, which can be interpreted as the separation between folds, has been kept fixed. It is not possible to consider it as an unknown and minimize the total energy density with respect to $L$ as well because it appears in the denominator of all the terms, see Eqs. (20,21,23), and therefore the optimal separation tends to infinity. Physically, we hypothesize that the separation is set by frictional forces between graphene and its substrate. Frictional forces in graphene

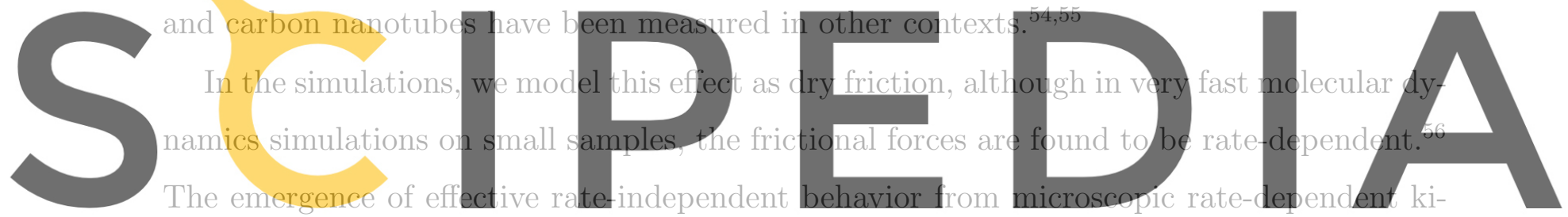

netics on complex energy landscapes has been studied theoretically in related contexts. ${ }^{57}$ Register for free at https//www.scipedia.com to download the version without the watermark We assume that the normal traction exerted by the graphene sheet on the substrate is

constant and given by the attractive adhesion forces. With this hypothesis, the tangential traction exerted by the substrate on the graphene sheet and opposing sliding is bounded by a constant traction $\tau_{0}$, which we view as a material parameter. Let us discuss now the treatment of friction in the analytical model. Since folding requires significant sliding of the graphene sheet relative to the substrate in a large fraction of the sample, see Figure 3(a) for an illustration, we assume that the whole surface undergoes sliding, which results in a simple expression for the work of dry friction

$$
W_{f}=\int_{\Omega} \tau_{0}|u| d S=\frac{1}{4} \tau_{0} \varepsilon_{0} L^{2} H
$$

where $H$ is the width of the sample. To systematically explore the separation, we consider a single fold in a sample of length $L$, and minimize the energy deviation per unit surface

$$
\Delta \bar{U}(A, B, l, L)=\Delta \bar{U}_{s}+\bar{U}_{b}+\bar{U}_{v}+\frac{1}{4} \tau_{0} \varepsilon_{0} L
$$


with respect to all its arguments. Since the last term is an increasing function of $L$ while the other terms are decreasing functions of $L$, this energy leads to a finite separation between folds.

\section{RESULTS AND DISCUSSION}

To check the consistency between the simulations and the theoretical model, we compare the results reported in Fig. 1(a-c) with the theoretical predictions (here, $\gamma=0.45 \mathrm{~J} / \mathrm{m}^{2}$ and $\left.h_{0}=6 \mathrm{~nm}\right)$. At a nominal strain of $\varepsilon_{0}=\left(L-L_{0}\right) / L_{0}=-0.4 \%$, wrinkling is the observed state for both models. In the simulations, the amplitude and wavelength of the wrinkles are $A \sim 0.09 \mathrm{~nm}$ and $\lambda \sim 5.5 \mathrm{~nm}$, to be compared with the theoretical model predictions, $A=0.090 \mathrm{~nm}$ and $\lambda=5.75 \mathrm{~nm}$. At a strain of $-0.7 \%$, the folding parameters measured from the simulation are $A \sim 0.65 \mathrm{~nm}, B \sim 0.48 \mathrm{~nm}$ and $l \sim 5.2 \mathrm{~nm}$, while the corresponding analytical predictions are $A=0.678 \mathrm{~nm}, B=0.423 \mathrm{~nm}$ and $l=5.14 \mathrm{~nm}$. Thus, the agreement between theory and simulations is excellent.

We consider now the behavior of graphene deposited on silicon oxide, as substrate with a well-characterized interaction potential. Reports indicate that the adhesion energy is on the order of $\gamma=0.45 \mathrm{~J} / \mathrm{m}^{2}$, and the equilibrium separation $h_{0}=0.6 \mathrm{~nm} \cdot{ }^{18,58}$ Unlike the response reported in Fig. 1(a-c) for a significantly larger equilibrium separation, wrinkling is not expected to be observable for graphene on $\mathrm{SiO}_{2}$. Indeed, the critical strain for wrinkling is estimated as $-1.64 \%$ by Eq. (11), while the wrinkle-to-fold strain is found to be $-1.71 \%$. Thus, the wrinkling regime is very narrow. At the wrinkle-to-fold strain, the amplitude of the wrinkles is, according to Eq. (15), $A=0.015 \mathrm{~nm}$. Such a small amplitude may explain why wrinkling morphologies have not been reported in supported graphene. It should be noted that, while the wrinkling strain is independent of the sample size $L$, the wrinkle-tofold strain is not. In fact, as the sample size (or separation between folds) becomes larger, the wrinkle-to-fold strain becomes closer to the critical wrinkling strain, further emphasizing our argument.

The adhesion energy between graphene and different substrates has been shown to significantly vary, from $0.72 \mathrm{~J} / \mathrm{m}^{2}$ for copper, ${ }^{19}$ to 0.07 for Polydimethylsiloxane (PDMS). ${ }^{6}$ More detailed features about the precise nature of the interaction potential between graphene and different substrates, such as the equilibrium separation or the inflection point, are not fully 


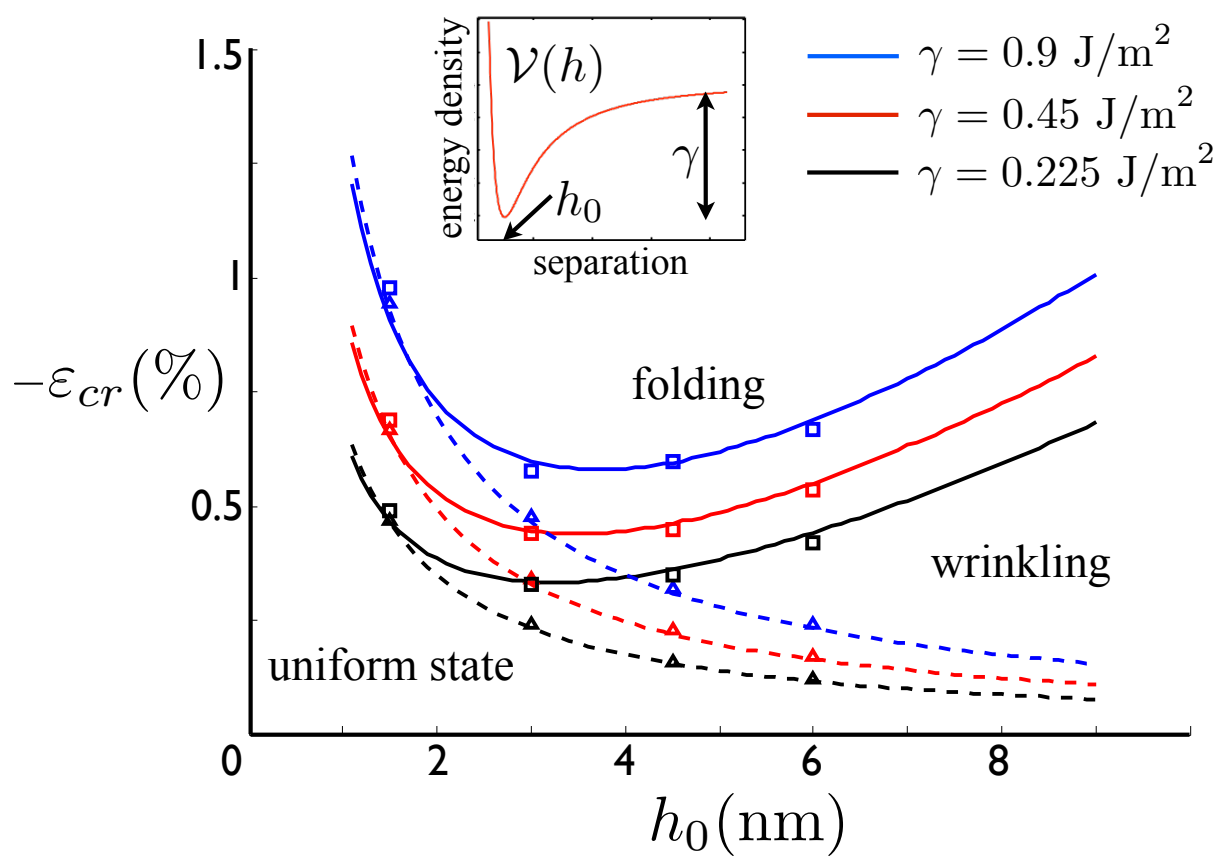

FIG. 2. Critical strains for the onset of wrinkling and the wrinkle-to-fold transition under uniaxial compression, for different van der Waals parameters and for a graphene film of length $L=200$ nm. Comparisons between analytical model (lines) and numerical simulations (symbols) for both wrinkling (dashed lines, triangles) and wrinkle-to-fold (solid lines, squares) strains as a function of the equilibrium spacing of the interaction potential $h_{0}$, and the adhesion energy $\gamma$ (different colors). The inset shows the features of the interaction potential.

characterized. It should also be noted that, in the presence of surface roughness, the effective interaction may differ from the intrinsic interaction. ${ }^{20}$ This suggests that parameters such as $\gamma$ and $h_{0}$ can vary significantly depending on the nature of the substrate, including its chemistry, its topography, or the environment conditions. Since transfer methods make it possible to deposit graphene on virtually any substrate, the graphene-substrate interaction potential may be tuned to a large degree with proper characterization. We examine in Fig. 2 the systematic dependence of wrinkling and folding on the interaction parameters $\gamma$ and $h_{0}$. Here, we fix $L$ and leave the effect of friction for later. The wrinkling strain is estimated by Eq. (11) and the wrinkle-to-fold strain by energy comparison with the wrinkled state. The theoretical model predictions (solid and dashed lines) are compared with simulations (symbols), obtaining an excellent agreement throughout the parameter space. In the simulations, we observe that folding is always preceded by wrinkling. For small values of $h_{0}$, the gap 
between these two strains becomes very small, and therefore, folding appears to bifurcate directly from the planar state. The theoretical model for folding is not very accurate for small $h_{0}$. In this regime, even at the onset of folding, the film deviation is significant and reaches beyond the point where the Taylor series expansion of the potential is accurate. While the critical wrinkling strain exhibits a monotonic decrease as a function of $h_{0}$, the wrinkle-to-fold strain attains a minimum for a finite equilibrium separation, which results in a broader wrinkling regime as $h_{0}$ becomes larger. As mentioned previously, the strain gap between the onset of wrinkling and the wrinkle-to-fold transition decreases as $L$ increases. Not surprisingly, the critical strains increase with adhesion energy.

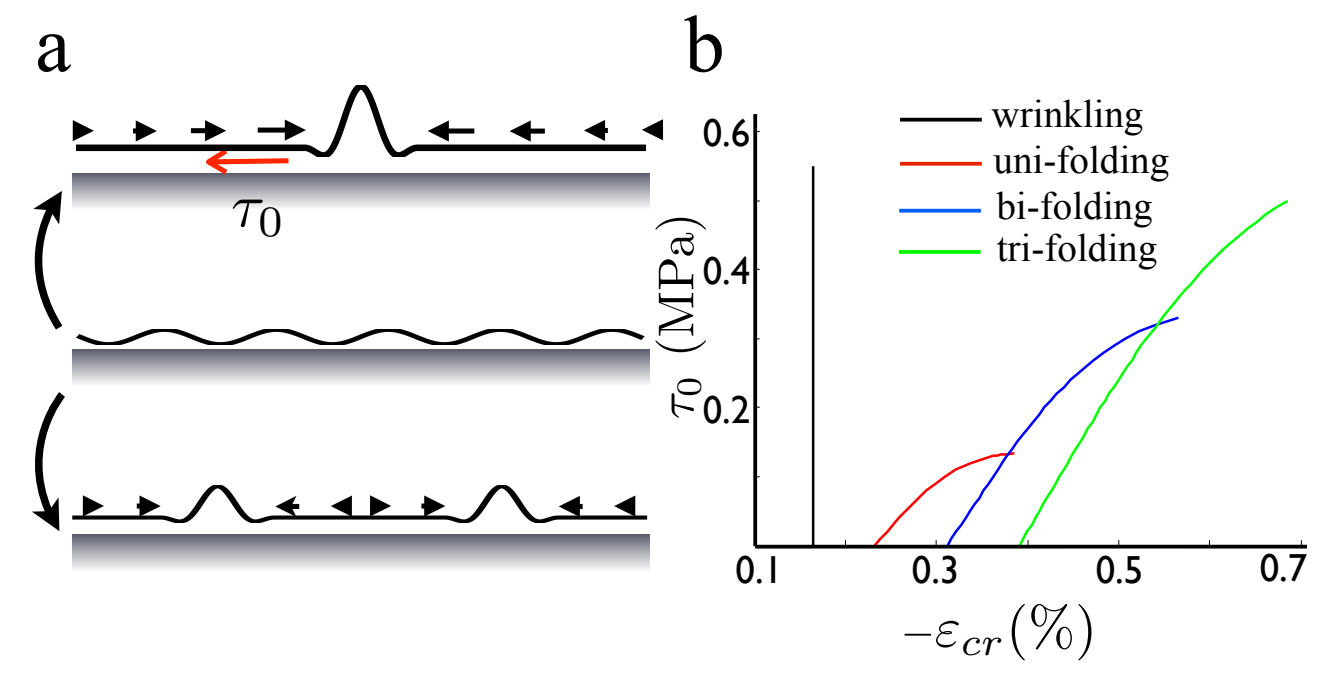

FIG. 3. Illustration of the sliding of graphene as the system transits from wrinkling, with distributed excess graphene area relative to substrate area, to folding, with localized excess area (a). Critical strain for wrinkling and for the transition from wrinkling to multiple folds as a function of the frictional parameter $\tau_{0}$, for a uniaxially compressed graphene sheet of length $L=1 \mu \mathrm{m}$ (b).

As introduced in Section III C, friction between graphene and the substrate can provide a selection principle for the fold separation. Indeed, as illustrated in Fig. 3(a), transitioning from a wrinkled state to a folded state requires sliding of the graphene sheet relative to the substrate, as the uniformly distributed excess area of a wrinkled configuration is brought to a localized fold. For a given applied strain, folds separated by a large distance are energetically favorable as compared to smaller folds closer to each other, but dissipate a larger frictional work. Thus, a competition between potential energy (elastic and adhesion) and frictional work is established. Although the tribological properties of supported graphene have been 
examined experimentally with AFM, ${ }^{55}$ no studies to our knowledge have characterized directly the graphene-substrate tribology. However, the frictional traction between different shells in multi-walled carbon nanotubes has been reported to be in the range $\tau_{0}=0.2$ to $0.85 \mathrm{MPa} .{ }^{54,59,60}$ These experiments probe a similar situation as that studied here, where the normal force confining the sliding surfaces is not externally applied but rather due to their cohesion. As mentioned earlier for the adhesion parameters, friction can be presumably controlled by the chemistry and topography of the substrate surface.

Figure 3(b) shows the critical wrinkling strain together with the critical strain for forming one, two, or three folds, as a function of the frictional parameter $\tau_{0}$ (the maximum shear traction that the substrate can exert on the graphene sheet) for a sheet of length $L=$ $1 \mu \mathrm{m}$. The adhesion parameters are $\gamma=0.45 \mathrm{~J} / \mathrm{m}^{2}$ and $h_{0}=4.5 \mathrm{~nm}$. We assume that wrinkling results in negligibly small tangental displacement, and thus the wrinkling strain is independent of $\tau_{0}$. In the absence of friction, the figure shows that configurations with a single fold are preferred. However, the work of friction affects in a different way the uni-, bi-, and tri-folding configurations, and for larger $\tau_{0}$, two or three folds become favorable.

We examine the systematic dependence of the fold separation as a function of frictional coefficient $\tau_{0}$ in Fig. 4, where we minimize the potential energy and frictional work in Eq. (26) with respect to $L$ as well. We test the theoretical model predictions against simulations for four selected values of $\tau_{0}$, for which 1,2, 3 and 7 folds are theoretically expected for a slab of length $1 \mu \mathrm{m}$. We find a very good agreement, although for the higher friction the simulation produces 6 folds instead of 7 . In fact, we find that for high friction, the number of fold can vary depending on the simulation details, e.g. numerical tolerance or size of the load steps, but always close to the number predicted by the theory. For such a nonlinear system with many metastable equilibria, this variability is not surprising. We also observe that the folds have uneven sizes (color indicates height, $w(x)$ ). Although Eq. (26) predicts that the fold separation decreases with the applied strain $\varepsilon_{0}$, the simulations show that the location of the folds is determined at the wrinkle-to-fold transition. As strain is further increased, the folds grow but stay laterally immobile, and we occasionally observe the disappearance of a smaller fold. The figure shows a sharp decrease in the fold separation as friction increases, which does not follow a power-law (inset). This non-trivial behavior is partially due to the fact that friction retards the wrinkle-to-fold transition strain in a complex way, Fig. 3(b). We find that the $\tau_{0}-L$ relationship weakly depends on the adhesion energy $\gamma$ as $L \propto \gamma^{a}$, where 


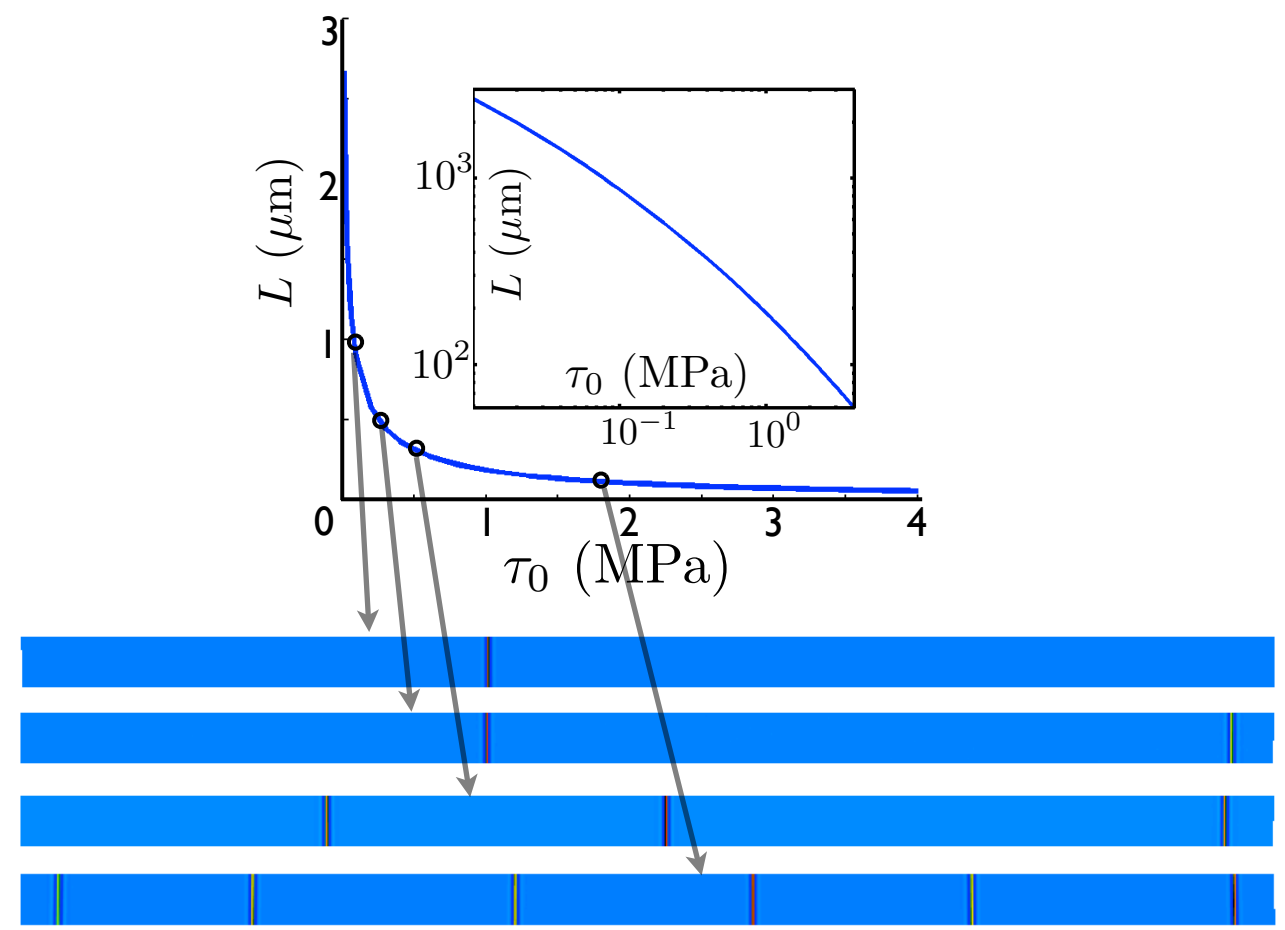

FIG. 4. Separation between folds $L$ as a function of the friction parameter $\tau_{0}$ predicted by the theoretical model (top), checked against the simulations for four selected frictional coefficients (bottom). The color represents the out-of-plane deviation $w(x)$. The inset shows $L$ as a function of $\tau_{0}$ in a log-log scale, to highlight the fact that the relation is not a power-law.

$0.2<a<0.43$ depends on $\tau_{0}$. The equilibrium separation $h_{0}$ plays a significant role for very small $\tau_{0}$. As noted earlier, in graphene samples grown by CVD the typical separation between the folds generated upon cooling is between hundreds of nanometers and microns, suggesting small frictional coefficients in the order of $\tau_{0} \approx 0.1 \mathrm{MPa}$. However, it should be noted that folding in CVD-grown graphene may take place at high temperatures, which in turn may decrease $\tau_{0}$ relative to the value at room temperature.

\section{SUMMARY AND OUTLOOK}

Inspired by observations of CVD-grown graphene and an increasing interest in controlling experimentally the out-of-plane morphology of supported graphene, we have examined by fully nonlinear simulations and by a theoretical model the mechanics of out-of-plane relaxation of graphene adhered to a rigid substrate and subjected to lateral uniaxial strain. We 

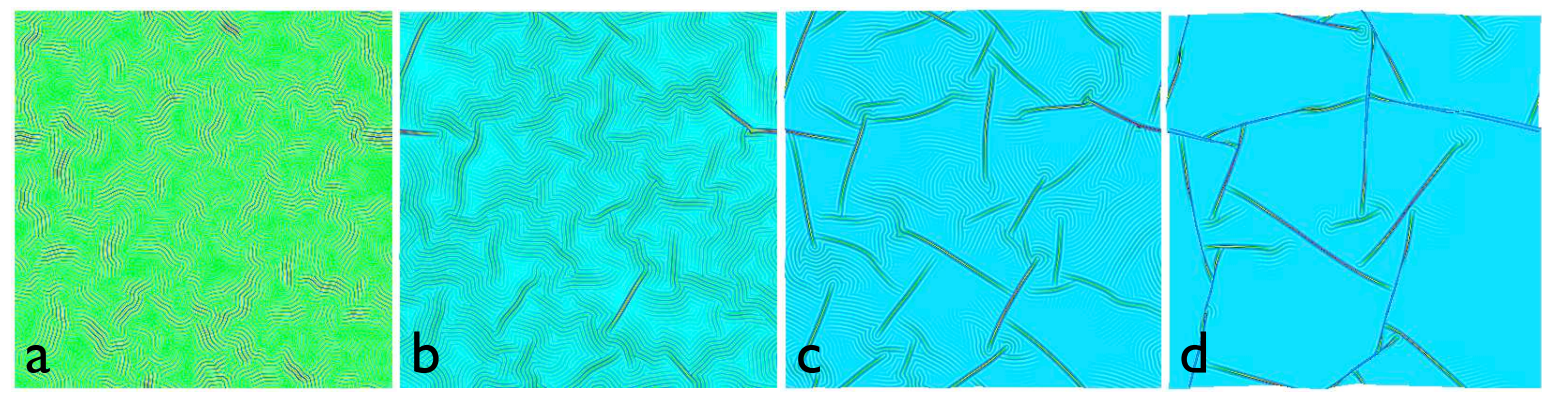

FIG. 5. Different stages during the wrinkle-to-fold transition of supported graphene upon biaxial compression for a $500 \mathrm{~nm} \times 500 \mathrm{~nm}$ sample. (a) Randomly distributed labyrinth wrinkles; (b) nucleation of short folds; (c) extension of folds at the expense of wrinkles; (d) network of folds. The areal strain $\Delta A / A_{0}$ is -0.01 in (a) and -0.04 in (d).

show that localized folds are preceded by wrinkling, although its small amplitude makes its experimental observation very difficult for common graphene-substrate interaction parameters, e.g. those in silicon oxide. While wrinkling is a rather generic process for many different systems, the transition to folding is a nonlinear phenomenon with different physical origins depending on the system. For supported graphene, folding results from the nonlinear nature of the interaction with the substrate, which softens and looses cohesion at large separations, thus promoting the emergence of large localized folds. We have analyzed the systematic dependence of the onset of wrinkling and of the wrinkle-to-fold transition on the adhesion energy and the equilibrium separation between graphene and the substrate. In the absence of nonlocal elastic fields in the substrate coupling different folds, ${ }^{36}$ we show that friction opposing sliding between graphene and the substrate selects the separation between folds.

We have found that the onset of folding and the fold spacing can be controlled by the adhesion and frictional properties between graphene and the substrate. It has been established experimentally that some of these properties can present large variations depending on the substrate material, e.g. silicon oxide, copper, or PDMS. Presumably, the chemistry and roughness of the surface of the substrate offer significant flexibility to tune these parameters. As illustrated in Fig. 5, the physics of wrinkling and folding under biaxial compression are more complex, as found in related systems. ${ }^{34}$ Domains with different wrinkling orientations initially form (a). Then, short folds nucleate throughout the domain, which partially release parallel wrinkles in their vicinity (b). These folds then grow (c) at the expense of wrinkles, and connect forming a network that delimits cells devoid of noticeable wrin- 
kles (d). Upon further compression, the network progressively reorganizes by simplifying the fold junctions. The resulting network closely resembles the fold morphologies observed experimentally. We are currently studying the control of these networks by the adhesion and tribological properties of the graphene-substrate interface and by the anisotropy of the strain. Thus, our findings and further work on biaxially strained graphene could provide guidance for strain-based engineering of graphene fold networks.

\section{ACKNOWLEDGMENTS}

We acknowledge the support of the European Research Council under the European Community's 7th Framework Programme (FP7/2007-2013)/ERC grant agreement nr 240487. KZ acknowledges the support of the UPC. MA acknowledges the support received through the prize "ICREA Academia" for excellence in research, funded by the Generalitat de Catalunya.

\section{REFERENCES}

${ }^{1}$ J. C. Meyer, A. K. Geim, M. I. Katsnelson, K. S. Novoselov, T. J. Booth, and S. Roth, "The structure of suspended graphene sheets," Nature, 446, 60 (2007).

${ }^{2}$ J. S. Bunch, A. M. van der Zande, S. S. Verbridge, I. W. Frank, D. M. Tanenbaum, J. M. Parpia, H. G. Craighead, and P. L. McEuen, "Electromechanical resonators from graphene sheets," Science, 315, 490 (2007).

${ }^{3}$ C. Lee, X. Wei, Q. Li, R. Carpick, J. W. Kysar, and J. Hone, "Elastic and frictional properties of graphene," Phys. Status Solidi B, 246, 2562 (2009).

${ }^{4}$ The nominal thickness is given by $h=\sqrt{12 D / Y_{s}}$, where $Y_{s}$ is the in-plane surface Young's modulus with units of line tension and $D$ the bending stiffness of graphene ${ }^{38,45}$.

${ }^{5}$ T. Li and Z. Zhang, "Substrate-regulated morphology of graphene," Journal of Physics D: Applied Physics, 43, 075303 (2010).

${ }^{6}$ S. Scharfenberg, N. Mansukhani, C. Chialvo, R. L. Weaver, and N. Mason, "Observation of a snap-through instability in graphene," Applied Physics Letters, 100, 021910 (2012). ${ }^{7}$ F. Guinea, B. Horovitz, and P. Le Doussal, "Gauge field induced by ripples in graphene," Phys. Rev. B, 77, 205421 (2008). 
${ }^{8}$ D. Rainis, F. Taddei, M. Polini, G. León, F. Guinea, and V. I. Fal'ko, "Gauge fields and interferometry in folded graphene," Phys. Rev. B, 83, 165403 (2011).

${ }^{9}$ W. Zhu, T. Low, V. Perebeinos, A. A. Bol, Y. Zhu, H. Yan, J. Tersoff, and P. Avouris, "Structure and electronic transport in graphene wrinkles," Nano Letters, 12, 3431 (2012).

${ }^{10} \mathrm{~K} . \mathrm{Xu}, \mathrm{P} . \mathrm{Cao}$, and J. R. Heath, "Scanning tunneling microscopy characterization of the electrical properties of wrinkles in exfoliated graphene monolayers," Nano Letters, 9, 4446 (2009).

${ }^{11}$ D. Srivastava, D. W. Brenner, J. D. Schall, K. D. Ausman, M. Yu, and R. S. Ruoff, "Predictions of enhanced chemical reactivity at regions of local conformational strain on carbon nanotubes: kinky chemistry," The Journal of Physical Chemistry B, 103, 4330 (1999).

${ }^{12}$ W. Bao, F. Miao, Z. Chen, H. Zhang, W. Jang, C. Dames, and C. N. Lau, "Controlled ripple texturing of suspended graphene and ultrathin graphite membranes," Nat Nano, 4, $562(2009)$.

${ }^{13}$ V. M. Pereira and A. H. C. Neto, "Strain engineering of graphene's electronic structure," Physical Review Letters, 103, 046801 (2009).

${ }^{14}$ J. Zang, S. Ryu, N. Pugno, Q. Wang, Q. Tu, M. J. Buehler, and X. Zhao, "Multifunctionality and control of the crumpling and unfolding of large-area graphene," Nature Materials, in press (2013).

${ }^{15}$ W. H. Duan, K. Gong, and Q. Wang, "Controlling the formation of wrinkles in a single layer graphene sheet subjected to in-plane shear," Carbon, 49, 2107 (2011).

${ }^{16}$ X. Huang and S. Zhang, "Morphologies of monolayer graphene under indentation," Modelling and Simulation in Materials Science and Engineering, 19, 054004 (2011).

${ }^{17} \mathrm{~S}$. Ghosh and M. Arroyo, "An atomistic-based foliation model for multilayer graphene materials and nanotubes," Journal of the Mechanics and Physics of Solids, 61, 235 (2013).

${ }^{18}$ S. P. Koenig, N. G. Boddeti, M. L. Dunn, and J. S. Bunch, "Ultrastrong adhesion of graphene membranes," Nat Nano, 6, 543 (2011).

${ }^{19}$ T. Yoon, W. C. Shin, T. Y. Kim, J. H. Mun, T.-S. Kim, and B. J. Cho, "Direct measurement of adhesion energy of monolayer graphene as-grown on copper and its application to renewable transfer process," Nano Letters, 12, 1448 (2012).

${ }^{20}$ J. Bunch and M. Dunn, "Adhesion mechanics of graphene membranes," Solid State Communications, 152, 1359 (2012), ISSN 0038-1098. 
${ }^{21}$ X. Li, W. Cai, J. An, S. Kim, J. Nah, D. Yang, R. Piner, A. Velamakanni, I. Jung, E. Tutuc, S. K. Banerjee, L. Colombo, and R. S. Ruoff, "Large-area synthesis of high-quality and uniform graphene films on copper foils," Science, 324, 1312 (2009).

${ }^{22}$ K. Kim, Z. Lee, B. D. Malone, K. T. Chan, B. Alemán, W. Regan, W. Gannett, M. F. Crommie, M. L. Cohen, and A. Zettl, "Multiply folded graphene," Phys. Rev. B, 83, 245433 (2011).

${ }^{23}$ A. W. Robertson, A. Bachmatiuk, Y. A. Wu, F. Schäffel, B. Büchner, M. H. Rümmeli, and J. H. Warner, "Structural distortions in few-layer graphene creases," ACS Nano, 5, 9984 (2011).

${ }^{24}$ A. N. Obraztsov, E. A. Obraztsova, A. V. Tyurnina, and A. A. Zolotukhin, "Chemical vapor deposition of thin graphite films of nanometer thickness," Carbon, 45 (2007).

${ }^{25}$ N. Liu, Z. Pan, L. Fu, C. Zhang, B. Dai, and Z. Liu, "The origin of wrinkles on transferred graphene," Nano Research, 4 (2011).

${ }^{26}$ Z. Pan, N. Liu, L. Fu, and Z. Liu, "Wrinkle engineering: A new approach to massive graphene nanoribbon arrays," Journal of the American Chemical Society, 133, 17578 (2011).

${ }^{27}$ V. E. Calado, G. F. Schneider, A. M. M. G. Theulings, C. Dekker, and L. M. K. Vandersypen, "Formation and control of wrinkles in graphene by the wedging transfer method," Applied Physics Letters, 101, 103116 (2012).

${ }^{28}$ F. Brau, H. Vandeparre, A. Sabbah, C. Poulard, A. Boudaoud, and P. Damman, "Multiple-length-scale elastic instability mimics parametric resonance of nonlinear oscillators," Nat Phys, 7, 56 (2011).

${ }^{29}$ Y.-P. Cao, B. Li, and X.-Q. Feng, "Surface wrinkling and folding of core-shell soft cylinders," Soft Matter, 8, 556 (2012).

${ }^{30}$ B. D. Leahy, L. Pocivavsek, M. Meron, K. L. Lam, D. Salas, P. J. Viccaro, K. Y. C. Lee, and B. Lin, "Geometric stability and elastic response of a supported nanoparticle film," Phys. Rev. Lett., 105, 058301 (2010).

${ }^{31}$ D. P. Holmes and A. J. Crosby, "Draping films: A wrinkle to fold transition," Phys. Rev. Lett., 105, 038303 (2010).

${ }^{32}$ E. Hohlfeld and L. Mahadevan, "Unfolding the sulcus," Phys. Rev. Lett., 106, 105702 (2011).

${ }^{33}$ D. Chen, S. Cai, Z. Suo, and R. C. Hayward, "Surface energy as a barrier to creasing of 
elastomer films: An elastic analogy to classical nucleation,” Phys. Rev. Lett., 109, 038001 (2012).

${ }^{34}$ P. Kim, M. Abkarian, and H. A. Stone, "Hierarchical folding of elastic membranes under biaxial compressive stress," Nat Mater, 10, 952 (2011).

${ }^{35}$ L. Pocivavsek, R. Dellsy, A. Kern, S. Johnson, B. Lin, K. Y. C. Lee, and E. Cerda, "Stress and fold localization in thin elastic membranes," Science, 320, 912 (2008).

${ }^{36}$ J. Zang, X. Zhao, Y. Cao, and J. W. Hutchinson, "Localized ridge wrinkling of stiff films on compliant substrates," Journal of the Mechanics and Physics of Solids, 60, 1265 (2012).

${ }^{37}$ Y. Wang, R. Yang, Z. Shi, L. Zhang, D. Shi, E. Wang, and G. Zhang, "Super-elastic graphene ripples for flexible strain sensors," ACS Nano, 5, 3645 (2011).

${ }^{38} \mathrm{M}$. Arroyo and T. Belytschko, "Finite crystal elasticity of carbon nanotubes based on the exponential cauchy-born rule," Physical Review B, 69, 115415 (2004).

${ }^{39}$ M. Arroyo and T. Belytschko, "Nonlinear mechanical response and rippling of thick multiwalled carbon nanotubes," Physical Review Letters, 91, 215505 (2003).

${ }^{40}$ S. Zhang, S. L. Mielke, R. Khare, D. Troya, R. S. Ruoff, G. C. Schatz, and T. Belytschko, "Mechanics of defects in carbon nanotubes: Atomistic and multiscale simulations," Phys. Rev. B, 71, 115403 (2005).

${ }^{41}$ J. Wu, K. C. Hwang, and Y. Huang, "An atomistic-based finite-deformation shell theory for single-wall carbon nanotubes," Journal of the Mechanics and Physics of Solids, 56, 279 (2008).

${ }^{42}$ I. Arias and M. Arroyo, "Size-dependent nonlinear elastic scaling of multiwalled carbon nanotubes," Phys. Rev. Lett., 100 (2008).

${ }^{43} \mathrm{M}$. Arroyo and T. Belytschko, "An atomistic-based finite deformation membrane for single layer crystalline films," Journal of the Mechanics and Physics of Solids, 50, 1941 (2002).

${ }^{44}$ M. Arroyo and T. Belytschko, "Finite element methods for the non-linear mechanics of crystalline sheets and nanotubes.” Int. J. Numer. Meth. Engng, 59, 419 (2004).

${ }^{45}$ Q. Lu, M. Arroyo, and R. Huang, "Elastic bending modulus of monolayer graphene," Journal of Physics D: Applied Physics, 42, 102002 (2009).

${ }^{46}$ C. Lee, X. Wei, J. W. Kysar, and J. Hone, "Measurement of the elastic properties and intrinsic strength of monolayer graphene," Science, 321, 385 (2008).

${ }^{47}$ L. A. Girifalco, M. Hodak, and R. S. Lee, "Carbon nanotubes, buckyballs, ropes, and a universal graphitic potential," Physical Review B, 62, 13104 (2000). 
${ }^{48}$ Z. H. Aitken and R. Huang, "Effects of mismatch strain and substrate surface corrugation on morphology of supported monolayer graphene," Journal of Applied Physics, 107, 123531 (2010).

${ }^{49}$ F. Cirak, M. Ortiz, and P. Schröder, "Subdivision surfaces: a new paradigm for thin-shell finite-element analysis," International Journal for Numerical Methods in Engineering, 47, 2039 (2000).

${ }^{50} \mathrm{~S}$. H. Im and R. Huang, "Wrinkle patterns of anisotropic crystal films on viscoelastic substrates," Journal of the Mechanics and Physics of Solids, 56, 3315 (2008).

${ }^{51} \mathrm{X}$. Chen and J. W. Hutchinson, "Herringbone buckling patterns of compressed thin films on compliant substrates," Journal of Applied Mechanics, 71, 597 (2004).

${ }^{52}$ Z. Y. Huang, W. Hong, and Z. Suo, "Nonlinear analyses of wrinkles in a film bonded to a compliant substrate," Journal of the Mechanics and Physics of Solids, 53, 2101 (2005).

${ }^{53}$ L. D. Landau and E. M. Lifshitz, Theory of Elasticity (Pergamon, 1959).

${ }^{54}$ B. Bourlon, D. C. Glattli, C. Miko, L. Forró, and A. Bachtold, "Carbon nanotube based bearing for rotational motions," Nano Letters, 4, 709 (2004).

${ }^{55}$ C. Lee, Q. Li, W. Kalb, X.-Z. Liu, H. Berger, R. W. Carpick, and J. Hone, "Frictional characteristics of atomically thin sheets," Science, 328, 76 (2010).

${ }^{56}$ E. H. Cook, M. J. Buehler, and Z. S. Spakovszky, "Mechanism of friction in rotating carbon nanotube bearings," Journal of the Mechanics and Physics of Solids, 61, 652 (2013), ISSN 0022-5096.

${ }^{57}$ R. Abeyaratne, C. Chu, and R. D. James, "Kinetics of materials with wiggly energies: theory and application to the evolution of twinning microstructures in a cu-al-ni shape memory alloy," Philosophical Magazine A, 457-497 (1996).

${ }^{58}$ W. Gao and R. Huang, "Effect of surface roughness on adhesion of graphene membranes," Journal of Physics D: Applied Physics, 44, 452001 (2011).

${ }^{59} \mathrm{~J}$. Cumings and A. Zettl, "Low-friction nanoscale linear bearing realized from multiwall carbon nanotubes," Science, 289, 602 (2000).

${ }^{60}$ M.-F. Yu, B. I. Yakobson, and R. S. Ruoff, "Controlled sliding and pullout of nested shells in individual multiwalled carbon nanotubes," The Journal of Physical Chemistry B, 104, 8764 (2000). 